\title{
INTERACCIONES SIGNIFICATIVAS PARA EL DESARROLLO HUMANO
}

\section{Significant interactions for human development}

\author{
Martha Llanos Zuloaga* \\ Relaciones Internacionales del Foro Paulo Freire
}

\begin{abstract}
Resumen
Para el desarrollo humano es vital el rol que cumple las interacciones tanto humanas como aquellas que involucran la naturaleza y objetos del contexto familiar, social y escolar. La presente es una reflexión sobre la importancia de las interacciones significativas de los adultos especialmente con los niños y niñas de modo que los aprendizajes cognitivos y socio emocionales producen un impacto en el desarrollo de los niños. El autor realiza una revisión después de 20 años del gran tema del niño interior del adulto y de las capacidades resilientes de niños y adultos forjadas y cimentadas en interacciones positivas

Dos décadas más tarde debemos añadir la tecnología digital, una intervención que cada día si bien ofrece algunos elementos positivos dentro del aprendizaje puede limitar enormemente las interacciones y comunicaciones cara a cara, limitar el potencial creativo y; las acciones de comunicación social con una exploración y curiosidad limitada a la acción pasiva.

En 1998 la pregunta ¿¿stán los niños del S XX actualmente padres, preparados para los niños del S XXI? sigue siendo válida y las reflexiones se amplían a como están preparados y cuanto escuchamos las voces de los niños del siglo XXI. En un siglo iniciado con grandes niveles de violencia, de consumismo y materialismo que nos llevan a abogar por mayores y mejores valores y desarrollo de conciencia para crear oportunidades valiosas de desarrollo de la niñez tanto en el Perú como a nivel Mundial.
\end{abstract}

Palabras clave: desarrollo humano, desarrollo infantil, resiliencia, niño interior, valores, interacciones significativas, derechos de niños, afectividad, voces de la infancia, juego, tecnología digital.

\begin{abstract}
For human development, the role of human interactions and those involving the nature and objects from family, social, and school contexts is vital. This study offers a reflection on the relevance of significant interactions between adults and children, in particular, in a way that cognitive and socioemotional learning has an impact on children's development. The author carries out a review after 20 years of the great topic of the adult's inner child and the resilient capabilities of children and adults forged and consolidated in positive interactions.

Two decades later, we must add the intervention of digital technology, which offers certain positive aspects for everyday learning, but can also considerably limit interaction and face-to-face communication, creative potential, and activities of social communication restricted to passive curiosity and exploration.

In 1998, the question: are the twentieth-century children, -nowadays parents- prepared for the twentyfirst century children? This is still a valid question today and the concern also includes to find out how well parents are prepared and how much the voices of twenty-first century children are heard. This century has begun with high levels of violence, consumerism and materialism, and requires to make a stand for greater and better values and awareness to create valuable opportunities for childhood development both in Peru and in the world.
\end{abstract}

Keywords: human development, childhood development, resilience, inner child, values, significant interactions, children's rights, affectivity, children's voices, play, digital technology.

\footnotetext{
* Psicóloga marthallanos@hotmail.com

ORCID: https://orcid.org/0000-0001-5456-1530
} 


\section{ANTECEDENTES}

El presente artículo se basa en las reflexiones actuales sobre un tema prioritario y relevante en el cual puse un énfasis particular en el momento en que culminábamos el siglo $\mathrm{XX}$ e ingresábamos con visiones para el XXI el nuevo milenio como le llamábamos. Fui seleccionada por La Organización Mundial de Educación Preescolar la OMEP para ser la Conferencista Principal y compartir una charla magistral en Dinamarca. Fue en el año 1998 que la OMEP celebrara sus bodas de oro de la más Antigua organización dedicada al bienestar de los niños menores de 5 años OMEP. Las reflexiones surgidas en el año 1998 en Dinamarca estaban vinculadas con la visión del rol de las interacciones y del crecimiento conjunto de Niños y adultos. En resumen, como podíamos vislumbrar a la niñez en este siglo XXI.

En mayo del 2019 y en el Perú celebramos 88 años de la educación inicial y el tema central se refiere a las interacciones significativas en la vida de los niños y niñas. Tema que me llevo a evocar y confrontar lo propuesto hacen dos décadas atrás y que siguen vigentes. El camino de experiencias recorridos en diversos países y continentes en estos 20 años a nivel mundial ha sido muy amplio, sin embargo, las situaciones de contexto que vivimos nos llevan a traer nuevamente la relevancia de estas interacciones y hacer un tributo a las múltiples profesoras, animadoras, promotoras y familias que han estado y están siempre al lado del niño escuchándolos y ofreciendo lo mejor de sí mismos para un desarrollo armónico e integral.

Teniendo como eje los derechos del niño y analizando en perspectiva lo propuesto en el año 98 reiteramos que el más grande derecho no escrito en ninguna convención es el derecho del niño a tener adultos especiales armónicos y respetuosos de sí mismos y de los niños, esos respetos unidos a una visión de dignidad hacen que en este siglo XXI aboguemos por escuchar las voces de los niños. Que se de paso a una escucha activa de las propuestas que los niños ofrecen y de las opiniones que nos lo muestran como un ser pleno de potencialidades.

En la conferencia del año 1998, exprese mi deseo de hacer un merecido homenaje a la libertad y autenticidad del niño y poder compartir y reflexionar acerca de la interacción de niños y adultos bajo una perspectiva actual y novedosa, basada en el rescate de las fuerzas positivas de los seres humanos, característica de nuestra misión para el siglo XXI.

El tema central del Congreso mundial que celebraba una memorable fecha, su edad dorada, era El derecho del niño al cuidado, juego y educación. En esta dimensión profundizamos la naturaleza propia del niño y las interacciones con los adultos haciendo énfasis en el tema de la resiliencia o capacidad de afrontar situaciones difíciles, capacidad de crecer en forma saludable (desde un punto de vista holístico) y dar mayor calidad y cualidad a la vida. Quise también aportar a la comprensión y fomento de la resiliencia (la palabra ha sido tomada directamente del inglés, resilience) con el análisis dinámico del "niño interior" del adulto, aspecto que cada día toma más fuerza, por representar la parte más auténtica, más integra y la más invencible, y veremos que solo en la medida en que el adulto conserva y recrea el niño interior sus posibilidades de creatividad, interacción, expansión y su propia resiliencia serán mayores.

Sumamente válida la pregunta que nos hicimos y era como está el adulto preparado para los niños cual es el contexto con el que se inicia el siglo XXI y ahora diremos estamos dos décadas más tarde.

\section{SIGLO XXI SITUACIÓN DE LA NIÑEZ EN LA REGIÓN}

El mundo actual se mueve entre extremos de conflictos y guerras, individualidad, materialismo, enfermedades y al mismo tiempo renace una búsqueda de valores y de un camino más espiritual que conlleve a mejorar la de vida de los seres humanos.

La mitad de la población menor de 18 años de la región, viven en condiciones de pobreza absoluta. Dentro de los grupos de alta vulnerabilidad están los niños y las niñas pobres de América Latina que están asistiendo a un deterioro progresivo de las condiciones de vida de sus grupos familiares: bajo nivel económico, escasa escolaridad, atraso en relación al tiempo que vivimos, exclusión del mercado, carencia de tierra y vivienda, asimismo el tráfico y consumo de drogas, grupos guerrilleros, migración del campo a la ciudad, 
delincuencia, inseguridad ciudadana, corrupción, discriminación de género, feminización de la pobreza, discriminación social, pauperización de los grupos indígenas, violación de los derechos humanos, atropello a las culturas nativas, discriminación de los discapacitados, discriminación de los ancianos, violencia intensificada contra niños y niñas y mujeres.

Si bien todos los países de la Región han ratificado la Convención sobre los Derechos del Niño (1989) y muchos han ajustado su legislación nacional a sus principios y mandatos, las reformas legislativas e institucionales nacionales se encuentran en proceso, generando estados de situación diversos y heterogéneos. La exigencia hoy en día, es la de reformular las políticas públicas, con un enfoque de derechos, que sea garantía, de protección integral. La planificación de Políticas Públicas para la Niñez y la Adolescencia en América Latina y el Caribe, debe contextualizarse en una región que enfrenta una crisis social y económica de difícil pronóstico.

Por otro lado, la pobreza aumentó más en aquellos hogares con mayor presencia de niños, niñas y adolescentes. El resultado de ello, es la alarmante tendencia hacia la "infantilización" de la pobreza.

El impacto que estas crisis han tenido en la Niñez, tiene como marco fundamental la destrucción de los núcleos familiares o la incapacidad de éstos, cuando existen, para atender las necesidades básicas y de Desarrollo Humano de sus miembros. El impacto ha ido tomando diversas expresiones y caracterizando diferentes rostros de la pobreza infantil: los niños y niñas callejeros, los niños y niñas drogadictos, los niños y niñas víctimas o protagonistas de la violencia familiar, los niños y niñas trabajadores, los niños y niñas víctimas de la prostitución infantil, las madres adolescentes, los niños y niñas afectados por el conflicto armado, los niños y niñas abandonados, los niños y niñas especiales, los niños y niñas desnutridos, los niños y niñas indígenas o de las comunidades negras discriminadas racialmente.

Una visión de vida centrada en el materialismo, el consumismo, la competencia, una necesidad inminente de adquisiciones, ha llevado a las personas a incrementar las adicciones al alcohol y drogas, así como los niveles de suicidio y esto especialmente en países en donde los niveles económicos están resueltos. El stress y la tensión produce, aun en gente joven, ataques de corazón, cáncer y otras enfermedades de nuestra época. ¿Ahora nos preguntamos el siglo XXI ya tiene cerca de 20 años qué reflexiones y valores beneficiaran a los niños?

Como están los niños de ayer del siglo XX, ¿preparados para los niños de hoy del siglo XXI? Escuchando las voces de la niñez

Relataba muy descriptivamente en el año 1998 como el niño luego de haber disfrutado plácidamente el navegar como delfín durante 9 meses llega a una situación en donde enmascarados (sistema de salud) en una sala de operaciones lo reciben y hasta posiblemente le den un palmazo por si no respira, así recibe la bienvenida al mundo. Actualmente si hablamos de derechos de niños su primer derecho es que su nacimiento sea una bienvenida una celebración y es así que recuerdo mi énfasis y aprecio a las culturas ancestrales en donde el nacimiento es toda una celebración comunitaria y en donde la naturaleza juega un rol muy importante se está acompañada de las dulas o amigas más cercanas y se le canta y la naturaleza se confabula para esta venida; siendo esta la primera interacción significativa.

La niñez es un mundo de posibilidades. La responsabilidad de los adultos en sus diferentes roles significa ejercicio de libertad. La base del desarrollo humano es la infancia, plena de potencialidades, la sociedad debe tener en cuenta al niño y la niña, reconociendo sus derechos. Esta es la educación del nuevo milenio, centrada en el ser, una educación biométrica, una delicada construcción con todas las inmensas potencialidades de que está dotada la niñez.

Iniciamos este nuevo milenio como un milenio de esperanza y con la convicción total de dirigir los esfuerzos mundiales hacia el fortalecimiento de la paz y la dignidad humana. Es así que la atención a la niñez se convierte en un pilar indiscutible de solidaridad humana. Es por ello que la educación inicial se ubica como la piedra angular del desarrollo humano.

El concepto de inteligencias múltiples (Gardner, 1983) ha permitido entonces comprender que la 
educación no se reduce a la escuela, sino que existen múltiples y variados espacios educativos de interacción con el niño y que por lo tanto los adultos tienen que tomar más conciencia de su rol en la interacción con los niños y niñas. La interacción es para mí base de un crecimiento conjunto de adultos y los niños y niñas.

Para muchos el niño o la niña son seres frágiles, requieren cuidado, amor, predomina su espontaneidad, expresan sus necesidades, se ríen sin motivo, anda explorando sus alrededores, gran observador, gran tejedor de fantasías, respiran armónicamente, ama a todos por igual, lloran y ríen, saltan y corretean, se inventan juegos, cantan y encantan, son transparentes, con una enorme fuerza interna.

Los adultos nos quedamos maravillados ante el coraje que muestran los niños y niñas, son capaces de afrontar situaciones muy difíciles y se ríen incluso de sus fracasos. Esto es porque la autenticidad e inocencia da coraje y claridad. Esto no es algo que se aprende o se fija como meta para lograr. Es algo innato en los pequeños. No hay pasado y no hay futuro es un presente.

La tarea de criar a los hijos encuentra hoy más dificultades y retos y por tanto requiere de mucha comprensión y apoyo. muy sutilmente se desvaloriza el rol de la crianza. madre y todos los educadores, asumen el deber de "enseñarle" e "instruirle". Saber escuchar a los niños y niñas es la base de las interacciones más significativas que pueden influir en su desarrollo posterior.

Muchos padres contribuyen a que el niño pierda o limite su propia creatividad, su identidad, cuando sus interacciones provienen más del miedo y de la inseguridad, de la utilización del poder de ser adulto con un rol y la percepción del niño como un ser totalmente indefenso.

¿Muchos padres se preguntan, cómo se puede mantener la cara original del niño?, la respuesta es simple: permite que puedas aprender de este niño.

La fuerza que él tiene y el amor que da a los demás te hará respetarlo, ¿pero si tienes miedo, cómo podrás cimentar, cuidar y favorecer el desarrollo de niños valientes y seguros de sí mismos? Una persona feliz es una persona no violenta y valiente y segura.

Los vínculos o llamado "apego" de las etapas iniciales de la vida son básicas para el desarrollo infantil ella conlleva; ser fomento de la seguridad básica del niño por ello estos vínculos incluyen elementos intra-psíquicos del niño y también un conjunto de procesos sociales (familia y red de apoyo externo); estas capacidades están en una dinámica de desarrollo progresivo, influenciadas por los cambios y las interacciones del niño en el ambiente; Se presentan como señala Kotliarenco (1998) como un estado y no una condición de ser.

Inicialmente el vínculo afectivo de los padres posibilita una interacción relajada de afecto tranquilizador, el padre se comunica de forma distinta a la madre ante su bebé, esta diferencia hace posible el acceso a los procesos cognitivos. Las bases de comunicación afectiva se dan partiendo del acercamiento y conocimiento reciproco de padres o adultos y niños.

Las figuras de apego influirán decisivamente en el desarrollo social del niño durante los tres primeros años de vida; El niño aprenderá a comunicarse afectivamente si la figura de apego ofrece una interacción adecuada es decir: rica y variada, constante y duradera, coherente a sus demandas y contextualizada en experiencias de aprendizaje, todo ello hace que se fundamente la seguridad emocional hacia la otra persona -amar y sentirse amado-, y hacia sí mismo -autoestima y conciencia positiva- que se traducen por ende en una conducta prosocial, donde aprenderá a vivenciar el estado emocional del otro, a tener autocontrol y mantener el carácter positivo de esas vivencias que transmite el vínculo afectivo (Cyrulnick, 2005).

Los vínculos basados en interacciones armoniosas y estables constituyen un punto crítico para el desarrollo infantil De allí que la presencia de una figura, aunque remota, estable y respondedora en la vida temprana del niño puede constituirse tanto en un factor protector que promueve un tipo de relación segura, contribuyendo así al fortalecimiento de la resiliencia en el niño. Desde este punto de vista, incluso los niños pequeños, tienen 
la capacidad de reconocer, diferenciar y aislar los modelos internos de trabajo de los padres (cuidadores primarios).

La "capacidad de reflexión" o conciencia crítica de los roles de los que establecen interacciones con los niños es muy poderosa. Ello por las posibilidades que tiene el cuidador de generar seguridad en el niño constituyéndose así en un factor protector especialmente poderoso en la transmisión de seguridad desde los padres (cuidadores primarios) hacia el niño.

El rol del juego en la vida de los niños es esencial, es su medio de expresión. Es lo lúdico que se ubica como lo más típico del niño, esa capacidad de recreación, de búsqueda de sensaciones, colores, expresiones, se da en una forma muy natural en el niño y le permite expresarse totalmente

Aquí es necesario llamar la atención al adulto que da al niño muchas "instrucciones", le habla de lo lúdico y de la expresión y hace de la silla un culto, prioriza reglas, y así va limitando el enorme potencial que tiene este niño. (lo dijimos en 1998 ahora nos referiremos posteriormente al culto a los "hipnotizadores" como les llamamos a los instrumentos tecnológicos)

La Resiliencia y sus Valores. ¿Por qué hablar de Resiliencia y vincularla con las interacciones con el Niño?

Las características del mundo de hoy requieren de un fortalecimiento de la resiliencia o la capacidad para dar respuesta, para permanecer en armonía, para tener un desarrollo más saludable aun en situaciones de adversidad, tenemos información sobre los factores que están contribuyendo a la resiliencia, Werner (1993) and Garmezy's (1991) refuerza las relaciones de confianza mutua; Loesel (1992) el factor emocional fuera de la familia; Wolin and Wolin (1993) el factor de autoestima; Segal (1989) la autonomía, la capacidad de tomar riesgos; Mrazek (1987) la sensación de ser amado; Osborn (1994), (Wang, Haertel \& Walberg 1997) indican el rendimiento escolar y desde 1979 aun sin mencionar dicha terminología Bronfrenbrenner (1979) ha contribuido decisivamente con el factor de amor incondicional. En el escenario de investigaciones en la década de los 80 la resiliencia era innovadora en su campo conceptual gracias a las investigaciones lideradas por Rutter, Grotberg, Vanistendal and Osborn; actualmente encontramos que el conocimiento acerca del tema de resiliencia ha sido ampliamente estudiado y documentado.

En América Latina encontramos nuevos desarrollos del concepto de resiliencia y es así como Suárez Ojeda (2001) nos plantea la resiliencia comunitaria la cual incluye los procesos de identidad cultural, la autoestima colectiva, la inclusión y el tema de la diversidad como una perspectiva de sólida identidad y que determina el aprecio por la riqueza de la diversidad y diferencia. Llanos (2009) retoma estos conceptos al desarrollar los vínculos de la resiliencia comunitaria en el desarrollo de las mujeres en comunidades de Asia y Latinoamérica.

La salud mental fue un factor clave al estudio de la resiliencia, ella se identificó con los sentimientos de seguridad del niño, con la confianza, de amor y la habilidad para resolver experiencias de adversidad.

Sin embargo, en la mayoría de los países el factor común es una limitada posibilidad personal y ayuda para afrontar la adversidad, lo cual sugiere que la promoción de la resiliencia debería ser incluida y para ello hay que revisar todos los postulados de interacción entre niños y adultos. Es por ello que el tema de interacciones ocupa un lugar muy importante.

El enfoque de la resiliencia es sumamente inspirador para nuestro trabajo y para nuestra vida personal. Nos permite releer nuestra historia personal, nuestras vinculaciones y aprender; nos permite tener una esperanza realista, que no elimina o niega los problemas, pero pone más atención en las fuerzas de la construcción.

La resiliencia mira a la vida como un proceso permanente de cambio, siendo su fuerza más permanente el amor y la aceptación incondicional. La resiliencia enfatiza la identificación de las redes formales e informales de apoyo. La resiliencia destaca los esfuerzos de los seres humanos a pesar de su analfabetismo, raza, color, ingreso, y prioriza la fuerza de la sabiduría para resolver positivamente situaciones. 
La resiliencia destaca la posibilidad de aprender de y con los niños y niñas y asumir responsablemente la aceptación de que toda situación y toda interacción encierra un mundo de mensajes y de aprendizaje.

En esta nueva mirada a las interacciones, y bajo la perspectiva de una respuesta para el siglo XXI, nos atrevimos a indicar que la llamada Educación de Padres y Adultos era muy necesaria, pero no a nivel de recetas hechas, sino como reflexión que llevara a crear una nueva visión más creativa y realista, basadas en la escucha consciente de las voces infantiles y de lo que siempre denomine que un derecho tácito de la niñez no escrito directamente en la Convención era interactuar con adultos cuyos valores y visión de la vida y la niñez permitiera unas interacciones que fruto eran las bases sólidas para el desarrollo humano.

En este sentido el Premio Nobel de economía Amartya Sen (1999) abogó siempre por la mirada que deberíamos tener sobre el niño y su contexto. El bienestar, la felicidad de los niños, su desarrollo armónico no es un reto proyectado al futuro, es un desafío presente, y los adultos tenemos que entender y asumirlos, no sólo desde la perspectiva de los derechos, sino desde la necesidad de transformar nuestras relaciones con los niños y las niñas cuyas vidas se encuentran afectadas por diversas problemáticas. Las investigaciones en calidad de vida pueden aportar conocimiento que contribuya a comprender desde los propios niños y niñas lo que esperan y requieren de sus padres, escuelas, del gobierno y de la sociedad

Las interacciones de niños y adultos tienen el potencial de creación conjunta, que permitiría a ambos alimentarse, mirarse en espejo y crecer psicológica y espiritualmente. Ambos podrían adquirir mayores y mejores herramientas para su desenvolvimiento, es pues una interacción de doble vía.

Las interacciones significativas juegan un rol muy importante en sentar las bases para una interacción positiva y significativa. En este sentido podemos traer el tema de pedagogía de la ternura como elementos especiales para el desarrollo armónico del niño con padres que respetan y conocen.
En un estudio sobre resiliencia realizado por la Fundación Van Leer (2002), en 4 países de América Latina se consideró como factores protectores individuales los atributos de la personalidad y los factores protectores internos, mencionando a continuación algunos de ellos:

- La espiritualidad, como una construcción del sentido de coherencia, en la vida cotidiana de las poblaciones indígenas extremadamente oprimidas (los Kollas en Argentina);

- La autoestima como factor protector de los niños negros (Brasil);

- La autoestima, autonomía, creatividad y el estado de ánimo como factores del desarrollo infantil (Brasil);

- El estado de ánimo como mecanismo de protección o de defensa en los niños, promovido a través de las técnicas circenses (Chile);

- La autoestima, la autonomía, la creatividad y el estado de ánimo, como factores protectores en la prevención del maltrato infantil (Perú);

Asimismo, se consideran como factores protectores del ambiente y factores protectores externos, las características familiares, sociales, físicas y culturales, tales como:

- Las relaciones afectivas (Argentina, Brasil, Chile y Perú);

- El apoyo social de una red de apoyo (Argentina, Brasil, Chile y Perú);

- La identidad étnica (Brasil);

- La identidad cultural (Argentina y Perú);

La promoción de la resiliencia es una tarea interdisciplinaria y la efectividad depende de la colaboración entre los diferentes niveles: político, institucional, comunitario, familiar e individual, siendo el objetivo de cada nivel, la promoción del bienestar del niño y una mejor calidad de vida para todos.

Los diversos autores señalan que, los niños resilientes muestran un vínculo seguro, y que éste último forma parte de un proceso que actúa como mediatizador en los comportamientos resilientes. 


\section{SIGLO XXI. LA ERA DIGITAL. LAS INTERACCIONES CON LA TECNOLOGÍA}

Esta etapa de la historia humana, la era digital, ya no exige que dos personas estén en la misma habitación (ni siquiera en el mismo país) para sostener una conversación. El ser humano ha llegado a desarrollar tecnologías y métodos de comunicación e interacción que se adaptan a medida que las opciones se vuelven más diversas y convenientes.

La gran pregunta especialmente vinculada al campo de las interacciones es: ¿está la comunicación digital reemplazando las interacciones cara a cara? hay quienes se han aventurado a asegurar que no se trata de una pregunta, sino de una realidad en el que el mundo está perdiendo cada vez más las interacciones humanas. (Levin, 2013)

Actualmente tenemos la creciente evidencia de los efectos negativos de la comercialización excesiva y la sobresaturación de medios sobre la salud, el desarrollo y las organizaciones de salud pública que muchos niños pasan demasiado tiempo frente a la pantalla, y que ésta se debería eliminar para infantes y limitar cuidadosamente para niños mayores.

Refiriéndonos al bienestar infantil y calidad de vida, de la niñez temprana se necesita estudiar la problemática en torno a las tecnologías de pantalla, tomar decisiones informadas sobre su uso en las aulas y centros de cuidado, y asesorar a los padres en la administración de contenido y tiempo frente a los medios tecnológicos.

Se comenta que, para niños mayores de 3 años, la exposición moderada a contenido de alta calidad educativa, sobre todo en compañía de un adulto que demuestre interés, puede promover el aprendizaje y la conducta pro-social. Por otro lado, algunos contenidos de pantalla pueden perjudicar a los niños. Está demostrado que los juegos y las actividades digitales que contienen un conjunto predeterminado de respuestas disminuyen la creatividad de los niños.

La exposición a la violencia en los medios de difusión está vinculada a la agresión, a la insensibilización a la violencia y a la falta de empatía para las víctimas. La violencia de los medios de comunicación también está asociada a la deficiencia en el rendimiento escolar.

¿Con esta tecnología qué lugar se deja para los juegos simbólicos, creativos y recreativos? Los niños pequeños y preescolares que pasan con las pantallas, menos tiempo se dedican a dos actividades esenciales para el desarrollo sano y el aprendizaje: el juego manual y creativo y la interacción con sus pares.

Las pantallas también les quitan tiempo para interactuar con adultos. Incluso cuando los padres miran televisión o vídeos con los niños, dedican menos tiempo a otras actividades con sus hijos. Además, los padres hablan menos con los niños cuando miran la televisión juntos o cuando la televisión esta de fondo. Las nuevas tecnologías también pueden interferir con la conversación entre padres e hijos y se vinculan a un déficit en niveles de comprensión.

Construyendo sobre lo positivo. Reflexiones para una nueva parentalizacion, compartiendo la alegría de crecer juntos

En el mundo de los símbolos, el niño representa la renovación, la divinidad, el entusiasmo vital, la capacidad de búsqueda, la esperanza, el futuro, la curiosidad, la valentía, la espontaneidad y la inmortalidad. Es el símbolo de la unión de todas las partes de la personalidad.

Es por ello que el niño interior es el alma de la persona, creada por medio de la experiencia vital. Según Carl Jung (2010) el niño representa una "plenitud" que abarca lo más profundo de la naturaleza". Posee el espíritu de la verdad, la espontaneidad y la autenticidad absoluta.

El niño interior del adulto constituyo en nuestra propuesta de interacciones en el año 98 el centro de nuestra reflexión retomando lo expresado indicamos que el niño interior ha estado siempre presente sin embargo se ha podido popularizar en los últimos treinta años. En la década de los setenta, la teoría y práctica de la educación se vieron conmovidas por las teorías de psicología infantil y teorías del desarrollo. Los aportes de Freud, Adler, Jung, Reich y otros respaldaron claramente lo que las tradiciones místicas habían valorado desde tiempos inmemoriales, 
el valor de la multitud de experiencias internas, siendo la infancia y la niñez las más importantes. Ha sido el psicólogo suizo Carl Gustav Jung, quien escribe sobre el arquetipo infantil, y describe al niño interior, como un símbolo de plenitud en la psique, como un puente entre el ámbito personal y el colectivo. (Llanos, 1998)

La comunicación afectiva se hace a través de los sentimientos y emociones mediada por los factores ambientales que influyen de manera decisiva en el desarrollo personal, puesto que es en el mundo exterior, el lugar donde encuentra el ser humano la realización o proyección de la satisfacción de sus necesidades fundamentales (Bronfenbrenner, 2002), entonces, una respuesta adecuada del ambiente favorece en el niño el equilibrio afectivo, la aceptación de la madre se expresa en una interacción positiva con su hijo, posteriormente esta identificación se llevará a cabo con el padre, de esta forma el niño encontrará la triada ambiental personificada que permitirá satisfacer sus necesidades primarias de tipo afectivo. (Cyrulnik, 2005).

En el ámbito familiar y de acuerdo con los enfoques sistémicos, las familias disfuncionales, causan daños incalculables a los hijos y al niño interior de sus miembros. El creciente reconocimiento del abuso infantil, indica que el niño interior maltratado puede ser el causante de abuso sobre su propia descendencia. $\mathrm{El}$ abuso infantil refleja siempre una falta de contacto y de respeto hacia el niño interior o psíquico.

A partir del establecimiento de los derechos de los niños, sin lugar a duda se han hecho esfuerzos para promover e intensificar la expresión de opiniones de los niños en las familias, las escuelas y en otras instituciones, para manifestar respeto frente a sus apreciaciones y propiciar su participación en los asuntos relacionados con su vida, como un derecho impostergable.

Inclusive, se han enmendado los códigos de procedimiento civil para asegurar que los niños y niñas sean escuchados en actuaciones judiciales que los afecten. Es necesario una mayor sensibilización para cambiar las actitudes tradicionales de los adultos que limitan o desaprueban la participación de los niños y niñas y de la verdadera inclusión de los niños en la formulación y evaluación de políticas, leyes y programas relacionados con su vida y para mejorar la calidad de vida de la población infantil, "tienen un profundo significado político en la medida en que deben abarcar la participación de los implicados en la evaluación de lo que los afecta" (Casas, 1998, p. 255), y en la elaboración de iniciativas para resolver las problemáticas o mejorar las situaciones en las que se desenvuelve su vida.

Las capacidades internas que debemos fortalecer en el niño se refieren a : la expresión y manejo de emociones, su autovaloración, autonomía, y sus capacidad de límites y control.

Las capacidades relacionales, están referidas a: la confianza, el humor, la cooperación, y el fortalecimiento de la identidad e integración cultural

Las condiciones necesarias de desarrollar en la familia son: atención de necesidades básicas y desarrollo de expresiones afectivas. Todo ello a través de interacciones significativas.

Hemos hecho un recorrido muy intenso en el mundo del niño y sus derechos, lo vemos como las raíces que están silenciosas debajo de la tierra creciendo y dando fuerza. Los niños tienen que ser cuidados y apoyados para que sean ellos mismos, cada niño es una versión única. Solo cuando una persona se ama a si misma puede dar lo mejor de sí y ser autentica. Primero se tiene que ser una luz para sí mismo y luego se irradiara a los demás.

En esta mirada retrospectiva y prospectiva queremos poner énfasis a los valores de adultos y niños. Los Valores juegan un rol primordial, hoy en día, Cooperación, Confianza en sí y en los demás, Honestidad, son elementos que los padres priorizan. Es muy importante que la valorización que de ellos hacen los contextos familiares y la escuela, presenten una consistencia armónica en lo que estos dos básicos núcleos de socialización e proponen en la educación de la niñez.

No hay nada más importante que criar a un niño, la diferencia entre criar y educar es arbitraria, pero en esta crianza es que hoy en día enfatizamos el 
desarrollo socioemocional del niño, con su sentido de seguridad, con su autoestima y el adulto o profesor asume el rol de facilitador o de animador en un proceso extraordinariamente interactivo y participativo.

La significación de la influencia parental, los factores individuales como los factores sociales y sus interrelaciones. La investigación actual en crianza se mueve desde estudios de microsistemas padres/ madres-niños, a transacciones con contextos sociales más amplios de escuela y cultura que nos remontan a traer nuevamente a Bronfenbrenner (1987) - en lo que llamó la ecología del desarrollo humano. La investigación actual de la crianza es guiada hoy por una perspectiva de bidireccionalidad entre factores biológicos y sociales, y ha tenido también un importante impacto tanto en la investigación como en la provisión de servicios en el campo de la intervención (Shonkoff $\&$ Meisels, 2000)

La tarea primordial será ayudar al niño a alcanzar mayor y mejor conciencia a ser más reflexivo. Niños y niñas están tratando de darse cuenta de los misterios de la vida y afrontan lo desconocido, su vida es una permanente aventura y exploración.

Los padres son los primeros que deben dar una mirada a su interior y recordar todo lo expuesto acerca del "niño interior y de los valores" y ser los primeros que busquen cambiar y mejorar. Si las personas no sienten a su niño interior como algo real, si no lo reconocen como una entidad existente, lo abandonan. ¿Si los adultos no son capaces de cambiar y de confiar en los demás como podrán ayudar a construir a la generación del siglo XXI? La gente hoy en día busca un enriquecimiento espiritual. El caos de la sociedad, la contaminación ambiental, la violencia, tienen su origen en la falta de confianza mutua.

El concepto de resiliencia invita a una pedagogía respetuosa con el niño, lo que representa la escuela, su significado tiene que ver con la aventura del conocimiento aunada a los lazos afectivos que entrelazan los adultos y sus pares. Ahora, los docentes no deben sobrevalorar la transmisión de conocimientos, sino que deben percatarse del poder del efecto de resiliencia con el vínculo afectivo pues es lo que verdaderamente enriquece el entorno del educando, enseñanza y aprendizaje favoreciendo el equilibrio riesgo-protección siguiendo a Cyrulnik (2003) "el significado de un objeto no se encuentra en el objeto, se encuentra en el entorno que atribuye un significado al objeto". (p. 106).

Teniendo como base que la resiliencia es la posibilidad de ser, amar y actuar, ella se vincula con personas que salen adelante, superan el traumatismo y continúan construyendo una vida que vale la pena ser vivida. Los educadores infantiles son los responsables de crear ambientes propicios de afecto, ternura y comprensión, teniendo en cuenta que no existe resiliencia sin traumas, y que el desamor, la violencia, la injusticia, la tragedia, no significa muerte y destrucción; seres heridos construyen respuestas creadoras de vida y saben avanzar a pesar de la adversidad (Cyrulnik, 2003). La resiliencia hace que "NINGUNA HERIDA SEA UN DESTINO". Boris Cyrulnik

Un avance muy valioso en estos 20 años recorridos es que aquello que propusimos como una visión del siglo XXI en el que los sistemas educativos deberían ensenar el arte de vivir, el arte de amar, el arte de compartir, de crear, de meditar, pero muchos sistemas lo han olvidado. Ahora desde el jardín de niños se "exige" aprendizaje de teoría, de conocimiento informativo, de rendimiento académico. Al llegar al final del siglo XX, el tema de valores está siendo analizado y redimensionado y conlleva una acción de reflexión colectiva. (Llanos,1998)

La responsabilidad significa libertad, poder volar sin alas, si se conoce la felicidad no se puede ser cruel con nadie, El trabajo de los adultos es muy delicado, la vida entera del niño está en sus manos. Deje que los niños se equivoquen, haga que se escuchen a sí mismos.

\section{REFLEXIONES FINALES}

Hemos querido compartir un largo recorrido a través del tiempo y de los elementos más importantes pilares en el desarrollo humano. El énfasis de la propuesta está en una visión amplia de la educación que conlleva la misión de la realización total del ser 
humano, no limitada a una visión instrumental, sino que trasciende a otras dimensiones de la persona.

Es así que la educación constituye un bien colectivo más allá del simple funcionamiento del mercado económico. La finalidad principal de la educación es el pleno desarrollo del ser humano, con sus dimensiones sociales, emocionales, espirituales. La educación es inherente y permanente a lo largo de toda la vida y todos los seres humanos sin distinción alguna y en forma incluyente somos agentes educativos a través de interacciones significativas.

¿Qué tipo de educación será necesario para el siglo XXI y para qué tipo de sociedad?

Es fundamental que retomando los Derechos del Niño es necesaria la existencia de adultos solidos con una visión de la niñez amplia con clara visión de las posibilidades y potencialidades de los niños y niñas. Por otro lado, en esta interacción los elementos brindados de consciente reflexión y desarrollo del propio niño interior del adulto, de los valores intrínsecos, dan poder a una dimensión más comprensiva y espiritual. En ello la calidad de las interacciones cara a cara, el cuidado, el juego, la participación infantil se constituyen en elementos básicos para un buen desarrollo infantil.

A diferencia de nuestra mirada y reflexiones en el artículo presentado en 1998 encontramos que un elemento que ha venido tomando mucho espacio en las interacciones es la tecnología y la pantalla de la tableta, del computador o TV se ha convertido en aquel elemento perturbador que interfiere en la comunicación cara a cara que limita la creatividad de la imaginación y del juego simbólico.

Debemos priorizar la reflexión de grupos de padres y madres de familia, de profesores, amigos y vecinos sobre el rol tan importantes de interacciones significativas que conllevan un aprendizaje y desarrollo socioemocional clave para la calidad de vida de los niños y niñas

\section{ESCUCHANDO A LA NIÑEZ*}

Martha Llanos

Para este mañana

La vida debería ser un campo de juego

el respeto la mejor religión

la evidencia de vida el estado de conciencia

el amor, el juego y el silencio lo prioritario

el sistema educativo basado en amor, libertad, respeto, oportunidades, virtudes

el pasado se ha centrado en conflictos

el futuro será centrado en amor

el pasado ha sido inconsciente

el futuro solo será consciente

no más políticas de la miseria

no más robots

aprendamos a decir que si

a la vida, al florecimiento

a nuestro niño interior y

a las interacciones significativas

que dejaran huellas en tu desarrollo humano.

La vida es un mundo de posibilidades

La vida está hecha de historias

Que la tuya sea autentica.

* Dedicado a la OMEP por su labor de 50 años en favor del desarrollo infantil. (Dinamarca 1998 revisada y recreada en 2019)

\section{BIBLIOGRAFÍA}

Bowlby, John (1973). Attachment and loss. Vol. 2, Londres: Penguin Books.

Bronfenbrenner, Uri (1987). La ecología del Desarrollo Humano. Experimentos en entornos naturales y diseñados. Barcelona: Paidós.

Bronfrenbrenner Uri,(1979) The Ecology of Human Development: Experiments by Nature and Design Cambridge, MA: Harvard University Press, 1979. 
Bronfrenbrenner, Uri (1979a). Contexts of child rearing: problems and prospects. American Psychologist, 34(10), 844-850.

Casas, F. (1998) Infancia: Perspectivas psicosociales. España: Grupo Planeta.

Casas, F. (2001). Video games: Between parents and children. En: I. Hutchby \& J. Moran Ellis: Children, technology and culture. The impacts of technologies in children's everyday lives, (pp. 4257). Londres: Routledge

Cyrulnik, Boris (1999). Un merveilleux malheur, París, Odile Jacob. [Traducción al español: La maravilla del dolor. El sentido de la resiliencia, Barcelona

Cyrulnik, Boris (2003). Los patitos feos, la resiliencia una infancia infeliz no determina la vida, $5^{\mathrm{a}}$ ed. Barcelona: Gedisa.

Cyrulnik, Boris, (2005) Bajo el signo del vínculo. España: Gedisa.

Fundación Van Leer (2002) Resiliencia en programas de desarrollo infantil temprano: estudio de revisión en cuatro programas de América Latina. Fundación Bernard van Leer.

Gardner, H. (1983) Frames of Mind: The Theory of Multiple Intelligences. New York: Basic Books.

Garmezy, N. (1991) Resiliency and vulnerability to adverse developmental outcomes associated with poverty. American Behavioral Scientist, 34, 416430 .

Grotberg. E. (1997) "La resiliencia en acción", trabajo presentado en el Seminario Internacional sobre Aplicación del Concepto de Resiliencia en Proyectos Sociales, Universidad Nacional de Lanús, Fundación Van Leer.

Jung, C. (2010) Psicología del arquetipo infantil. Obra completa. Volumen 9/1. 2da. Edición. Madrid: Trotta

Henderson, N. y Milstein, M (2003): Resiliencia en la escuela, Buenos Aires: Paidós

Kotliarenco, M. A., Cáceres, I. y Fontecilla, M. (1997) Estado de arte en resiliencia. OPS, Fundación W. K. Kellog, Agencia Sueca de Cooperación Internacional para el Desarrollo, CEANIM.
Levin, Diane (1998). Remote control childhood?: combating the hazards of media culture. Washington, D.C: National Association for the Education of Young Children.

Levin, Diane E. (2013) Beyond remote-controlled childhood: teaching young children in the media age. Washington, D.C: National Association for the Education of Young Children.

Löesel, Friedrich (1992). Resilience in Childhood and Adolescence. Ginebra: International Catholic Child Bureau.

Llanos, M. (1998). Compartiendo la alegría de crecer juntos. Niños y adultos. International Journal of early childhood. October 30 (2), 8-18. Doi: 10.1007/BF03185630

Llanos, M. (2009). Resiliencia y género: promotoras comunitarias en Asia y América Latina. Temática psicológica: revista especializada de los programas académicos de Doctorado en Psicología, 5, 51 - 58. Perú

Melillo, A. \& Suarez, N. (comps.) (2001). Resiliencia: Descubriendo las propias fortalezas. Buenos Aires: Paidós.

Mrazek, P. J., \& Mrazek, D. A. (1987). Resilience in child maltreatment victims: A conceptual exploration. Child Abuse \& Neglect, 11(3), 357366.

Naciones Unidas (1989) Convención de los derechos de los niños. https://www.un.org/es/events/ childrenday/pdf/derechos.pdf

Osborn, A. (1994). Resiliencia y estrategias de intervención. Ginebra: BIC

Rutter, Michael (1987). Psychosocial resilience and protective mechanisms. American Journal Orthopsychiatry, 57 (3), 316-329

Sen, A. (1999). Invertir en la infancia: su papel en el desarrollo. Conferencias magistrales. Banco Interamericano de Desarrollo. Departamento de Desarrollo Sostenible. División de Desarrollo Social.

Shonkoff, J. P., \& Meisels, S. J. (Eds.). (2000). Handbook of early childhood intervention, 2nd ed. New York: Cambridge University Press. 
Segal, M. W. (1989). The nature of work and family linkages: A theoretical perspective. In G. L. Bowen \& D. K. Orthner (Eds.), The organization family: work and family linkages in the U.S. military. New York: Praeger.

Suárez Ojeda, E.N. (2001). Una concepción latinoamericana: la resiliencia comunitaria. En A. Melillo y E. N. Suárez Ojeda. Resiliencia: descubriendo las propias fortalezas. Barcelona: Paidós.

Vanistendael, S. (1994). Cómo crecer superando los percances. Resiliencia: capitalizar las fuerzas del individuo. Ginebra: BICE
Werner, Emmy E. (1993). Protective factors and individual resilience. En: Handbook of early childhood intervention (1993) Meisels, Samuel J. Y Shonkoff, Jack P. (Eds.). Cambridge University Press. Nueva York, E.E.U.U.

Wolin, S. \& Wolin, J. (1993). The resilient self. New York: Villard Books.

Fecha de recepción: 9 de octubre, 2019

Fecha de aceptación: 7 de noviembre, 2019 